\title{
Unsupervised Clustering for Fault Diagnosis in Nuclear Power Plant Components
}

\author{
Piero Baraldi, Francesco Di Maio \\ Energy Department, Politecnico di Milano \\ Via Ponzio 34/3, 20133 Milano, Italy \\ E-mail: piero.baraldi@polimi.it,francesco.dimaio@polimi.it \\ Enrico Zio \\ Chair on Systems Science and the Energetic challenge \\ Ecole Centrale Paris and Supelec, France; \\ Energy Department, Politecnico di Milano \\ Via Ponzio 34/3, 20133 Milano, Italy \\ E-mail: enrico.zio@ecp.fr, enrico.zio@supelec.fr,enrico.zio@polimi.it \\ Received 30 June 2011 \\ Accepted 12 June 2012
}

\begin{abstract}
The development of empirical classification models for fault diagnosis usually requires a process of training based on a set of examples. In practice, data collected during plant operation contain signals measured in faulty conditions, but they are 'unlabeled', i.e., the indication of the type of fault is usually not available. Then, the objective of the present work is to develop a methodology for the identification of transients of similar characteristics, under the conjecture that faults of the same type lead to similar behavior in the measured signals. The proposed methodology is based on the combined use of Haar wavelet transform, fuzzy similarity, spectral clustering and the Fuzzy C-Means algorithm. A procedure for interpreting the fault cause originating the similar transients is proposed, based on the identification of prototypical behaviors. Its performance is tested with respect to an artificial case study and then applied on transients originated by different faults in the pressurizer of a nuclear power reactor.
\end{abstract}

Keywords: Fault diagnosis, unsupervised clustering, Haar wavelets, fuzzy similarity, spectral clustering, Fuzzy CMeans.

\section{Introduction}

Fault diagnosis can be seen as a classification problem in which a class identifying the type of the fault needs to be associated to a vector of values of measured signals [Zio et al., 2006a]. Due to the complexity of the phenomena involved and the highly non-linear interrelationships between the causes that determine the equipment behavior and the signal evolutions, it is usually difficult to develop analytical models for fault diagnosis [Venkatasubramian et al., 2003].

An attractive alternative is to resort to empirical classification models (classifiers) whose parameters are tuned through an iterative process, called training, based on a set of examples constituted by signals labelled with the corresponding class of fault under which conditions they have been measured [D'Antone, 1992; Reifman, 1997; Sheng et al., 2004; Zio, 2007; Zio et al., 2008]. Methodological approaches have been proposed for fault diagnosis in components of Nuclear Power Plants (NPPs) [Cheon et al., 1993; Kim et al., 1996; Reifman, 1997; Zio et al., 2006a; Zio et al., 2006b; Baraldi et al., 2010; Di Maio et al., 2011]. However, application in practice is limited because of lack of examples for classifier training. Indeed, although data collected during plant operation contain also signals measured in 
faulty conditions, the information on the fault class is usually not available.

The objective of this work is to develop a methodology for the identification of transients originated by faults of the same class, on the conjecture that they lead to similar behaviors of the measured signals. To this aim, the problem is formulated as one of clustering, in which the vectors of measured signal values are partitioned into a small number of homogeneous clusters so that those belonging to the same cluster are as similar as possible, and as dissimilar as possible to those belonging to the other clusters. In this work, the task of clustering of measured signals is addressed by means of a modified spectral clustering algorithm. The similarity between measured signals is computed by using a fuzzy similarity measure [Joentgen et al., 1999; Na et al., 2004; Zio et al., 2010; Zio et al., 2012] in the space of wavelet transforms [Strang et al., 1996; Ikonomopoulos et al., 1998] in order to account for the signals evolution in time (in the following also referred to as "trajectories"). A similarity graph is built, in which each vertex represents a trajectory and the weight associated to the edge connecting two vertices is the value of (fuzzy) similarity between the two corresponding trajectories. Spectral analysis techniques are finally applied in order to find an optimal partition of the graph [von Luxburg, 2007].

The proposed methodology has been tested on an artificial case and then applied to a case study concerning simulated faults in a pressurizer of a Pressurized Water Reactor (PWR) NPP.

The remaining of the paper is organized as follows: Section 2 states the problem; Sections 3.1 and 3.2 sketch the methodology proposed for unsupervised clustering of transients; Section 4 presents the artificial case study used to verify the performance of the proposed methodology; Section 5 presents the case study concerning the pressurizer of the PWR; finally, in Section 6 some conclusions and remarks are drawn.

\section{Problem statement}

Let us assume that the values of $Z$ signals at different times have been measured during $N$ plant transients originated by faults of different classes. In practice, the generic $i$-th transient can be seen as a trajectory into the $Z$ dimensional signal space and represented by the matrix of values $\overline{\overline{X^{i}}}$ whose component $x_{l k}^{i}$ represents the value of signal $k$ taken at time $t_{l}, k=1, \ldots, Z$ and $l=0, \ldots, T_{i}-1$, where $T_{i}$ is the number of available measurements for the $i$-th trajectory. For the sake of simplicity, in this work we assume that:

- transients begin at time $t_{0}$;

- signal measurements are taken at fixed time steps, $\Delta t ;$ thus, $t_{l}=l \cdot \Delta t$;

- all transients have the same duration $(T-1) \cdot \Delta t$, i.e., $T_{i}=T$, for any $i=1, \ldots, N$.

The objective of the present work is to partition the $N$ trajectories $\overline{\overline{X^{i}}}$ into an unknown number of clusters, $C$, each one containing transients characterized by similar behavior.

\section{Methodology}

The methodology here proposed is based on spectral clustering [Strang et al., 1996]. The main characteristic of spectral clustering is that it allows partitioning objects (in our case, vectors of measured signals) into clusters by using only a measure of similarity between them. A similarity graph $G=(V, E)$ is introduced, in which each vertex $v_{i}$ in this graph represents an object and a weight is associated to each edge $e_{i j}$ connecting vertices $i$ and $j$, to measure the similarity between objects $i$ and $j$ [von Luxburg, 2007]. Clustering then aims at finding a partition of the graph such that the edges between elements belonging to different groups of the partition have small weights (which means that objects in different clusters are dissimilar from each other) and the edges connecting elements within the same group have large weights (which means that objects within the same cluster are similar to each other) [Alpert et al., 1999]. Section 3.1 illustrates the method proposed to measure similarity between the trajectories, whereas Section 3.2 illustrates the details of the spectral clustering algorithm.

\subsection{Similarity measure between trajectories}

The notion of similarity is strongly related to the objective of the application: in our case, we want a similarity measure that takes large values for trajectories of the same class (transients caused by the same type of fault) and small values for trajectories of different classes.

When looking at the similarity between trajectories, the functional behaviour of the signals is the focus of the analysis irrespective of the numerical values which may be quite dissimilar due to the presence of outliers, noise or different scaling and translating factors 
[Angstenberger, 2001]. Possible causes of difference in the signal numerical values from transients of the same class are the magnitude of intensity of the faults, the plant operational state, the measurement noise. For this reason, the definition of the similarity measure between two transients should not be based on the magnitude of the signal values, but rather it should consider the functional characteristics of the signal trajectories, e.g., form, slope, curvature [Joentgen et al., 1999]. To catch these characteristics pre-processing of the transient data can be performed. Section 3.1.1 illustrates the data preprocessing technique applied in this work, whereas Section 3.1.2 defines the similarity measure adopted.

\subsubsection{Wavelet transform pre-processing}

Wavelet transforms have been chosen due to their effectiveness in catching the functional behaviour of the signals in problems of transient classification. [Roverso, 2000], [Roverso, 2003] and [Baraldi et al., 2012a] have shown the improved performance of transient classification algorithms when they are fed by wavelet features instead of the direct signal values. In the present work, the Haar wavelet transform [Ogden, 1997] is applied on a sliding window of the signal timeseries. For each signal $k=1, \ldots, Z$, the retained wavelet features are: the mean value, $w_{1}$, the maximum wavelet coefficient, $w_{2}$, and the minimum wavelet coefficient, $w_{3}$. The first feature is proportional to the average value in the time window and captures the general trend of the signal in the windows in a compact way. The features $w_{2}$ and $w_{3}$ capture variations in the signal within the window (e.g., downward or upward trends, step changes, spikes, etc.). The window size $T_{s}$ is selected so as to correspond to wavelet dyadic decomposition values (i.e., powers of 2), and consecutive windows are chosen with an overlap of $T_{s}-1$ to avoid missing features that might be present at the window borders.

Since three different wavelet features are obtained from each signal and given that the first transformation can be performed only when the first $T_{s}$ measurements are available, the matrix $\overline{\overline{X^{i}}}$ of size $[T-1, Z]$ is transformed into a matrix $\overline{\overline{Y^{i}}}$ of size $\left[T-T_{s}, 3 Z\right]$ which constitutes the new representation of trajectory $i$.

\subsubsection{Fuzzy similarity measure}

After the data pre-processing, the similarity between transient $i$ and transient $j$ can be computed by considering matrixes $\overline{\overline{Y^{i}}}$ and $\overline{\overline{Y^{j}}}$. A fuzzy similarity measure is considered, which determines the degree of closeness of the two trajectories with reference to the pointwise difference between the corresponding feature values [Zio et al., 2010]. In particular, the pointwise difference $\delta_{i j}$ between the $3 \cdot Z\left(T-T_{s}\right)$ values of trajectories $\overline{\overline{Y^{i}}}$ and $\overline{\overline{Y^{j}}}$ is defined by:

$$
\delta_{i j}=\sum_{k=1}^{3 Z} \sum_{l=1}^{T}\left(y_{k l}^{i}-y_{k l}^{j}\right)^{2}
$$

The similarity measure should allow for a gradual transition [Joentgen et al., 1999]. This is here achieved by evaluating the pointwise difference of two trajectories with reference to an "approximately zero" fuzzy set (FS) specified by a function which maps $\delta_{i j}$ into a value $\mu_{i j}$ of membership to the condition of "approximately zero": values of $\mu_{i j}$ close to 0 indicate that the signal evolutions in the two transients $i$ and $j$ are very different, whereas values close to 1 indicate high similarity.

Common membership functions can be used for the definition of the FS, e.g. triangular, trapezoidal, and bell-shaped [Dubois et al., 1988]. In the applications illustrated in this work, the following bell-shaped function is used:

$$
\mu_{i j}=e^{-\left(\frac{-\ln (\alpha)}{\beta^{2}} \delta_{i j}\right)}
$$

The arbitrary parameters $\alpha$ and $\beta$ can be set by the analyst to shape the desired interpretation of similarity into the fuzzy set: the larger the value of the ratio $-\ln (\alpha) / \beta^{2}$, the narrower the fuzzy set and the stronger the definition of similarity [Zio et al., 2010].

\subsection{Spectral clustering}

The computation of the fuzzy similarity between all possible pairs of trajectories originates the similarity matrix $\stackrel{W}{W}$ of size $[N, N]$, whose generic element $\mu_{i j}$ represents the fuzzy similarity between trajectories $i$ and $j$. The diagonal components $\mu_{i i}$ are set to 1 and the matrix is symmetric $\left(\mu_{i j}=\mu_{j i}\right)$.

From the matrix $\bar{W}$ a similarity graph $G=(V, E)$ is constructed, where each vertex $v_{i}$ represents the $i$-th trajectory and the weight associated to the edge $e_{i j}$ connecting the two vertices $i$ and $j$ is the similarity value $\mu_{i j}$ [von Luxburg, 2007]. The original problem of identifying groups of similar trajectories can be 
reformulated in that of finding a partition of the similarity graph such that the edges connecting elements of different groups have small weights and the edges connecting elements within a group have large weights [Alpert et al., 1999]. The spectral clustering algorithm is based on the following steps:

\section{Step 1: normalized Graph Laplacian Matrix}

Compute:

- the degree matrix $\overline{\bar{D}}$ which is a diagonal matrix with diagonal entries $d_{1}, d_{2}, \ldots, d_{N}$ defined by

$$
d_{i}=\sum_{j=1}^{N} \mu_{i j}, i=1,2, \ldots, N
$$

- the normalized graph Laplacian matrix:

$$
\overline{\bar{L}}_{s y m}=\overline{\bar{D}}^{-1 / 2} \overline{\bar{L}}^{-1 / 2}=\overline{\bar{I}}-\overline{\bar{D}}^{-1 / 2} \overline{\bar{W}} \overline{\bar{D}}^{-1 / 2}
$$

where $\overline{\bar{L}}=\overline{\bar{D}}-\overline{\bar{W}}$ and $\overline{\bar{I}}$ is the identity matrix of size $[N, N]$.

\section{Step 2: eigenvalues and eigenvectors of $\boldsymbol{L}_{\text {sym }}$}

The information on the structure of a graph can be obtained from its spectrum [Zhao et al., 2007]. Given $\overline{\bar{L}}_{s y m}$, compute the first $C$ eigenvalues $\lambda_{1}, \lambda_{2}, \ldots, \lambda_{C}$ and corresponding eigenvectors $\bar{u}_{1}, \bar{u}_{2}, \ldots, \bar{u}_{C}$. The first $C$ eigenvalues are such that they are very small whereas $\lambda_{C+1}$ is relatively large [von Luxburg, 2007].

\section{Step 3: Number of clusters}

The number of clusters is set equal to $C$, according to the eigengap heuristic theory [Mohar, 1997].

\section{Step 4: Feature extraction}

The relevant information on the structure of the matrix $\overline{\bar{W}}$ is obtained by considering the eigenvectors $\bar{u}_{1}, \bar{u}_{2}, \ldots, \bar{u}_{C}$ associated to the $C$ smallest eigenvalues of its laplacian matrix $\overline{\bar{L}}_{s y m}$. The square matrix $\overline{\bar{W}}$ is transformed into a reduced matrix $\overline{\bar{U}}$ of size $[N, C]$, in which the $C$ columns of $\overline{\bar{U}}$ are the eigenvectors $\bar{u}_{1}, \bar{u}_{2}, \ldots, \bar{u}_{C}$. Thus, the $i$-th trajectory similarity with other trajectories is captured in the $C$-dimensional vector $\bar{u}_{i}$ corresponding to the $i$-th row of the matrix $\overline{\bar{U}}$. A matrix $\bar{T}$ is formed from $\overline{\bar{U}}$ by normalizing its rows [von Luxburg, 2007]:

$$
t_{i c}=\frac{u_{i c}}{\left(\sum_{c=1}^{c} u_{i c}^{2}\right)^{0.5}}, i=1,2, \ldots, N, c=1,2, \ldots, C
$$

It has been shown that this change of representation enhances the cluster properties in the data, so that clusters can be more easily identified [von Luxburg, 2007].

\section{Step 5: Unsupervised clustering}

In this work, we resort to the Fuzzy C-Means (FCM) algorithm to partition the data into $C$ clusters [Bezdek, 1981; Leguizamon et al., 1996; Alata et al., 2008]. FCM originates from hard C-Means clustering: the difference is that it allows elements (trajectories, in our case) to belong to two or more clusters [Klir and Yuan, 1995]. For each $i$-th element, the algorithms provides its membership $m_{i c}$ to all clusters, $c=1,2, \ldots, C$. If needed, crisp assignment can be obtained, e.g., by considering the cluster to whom the element belongs with the largest membership value. A prototypical trajectory can be identified for each cluster by considering the trajectory with the largest membership value to the cluster. The analysis of such trajectories can guide understanding, identification and interpretation of the fault types.

\section{The artificial case study}

The performance of the methodology has been firstly verified with respect to an artificial case study built by simulating $N=150$ trajectories of $C=5$ different classes in a $Z=4$ dimensional signal space (Figures 1-4). Transient length $T$ is 100 time steps. Each of the 5 classes of transients is formed by 30 trajectories characterized by a priori established functional behaviours of the 4 signals (e.g., linear, parabolic and exponential). All the transients of the same class differ only for different values of the parameters governing the functional behaviour (e.g., the slope value of a linear functional behaviour) whereas two transients of different classes have at least one signal with a different functional behaviour [Baraldi et al., 2012b]. Since the information on the class of each trajectory and on the total number of classes is not expected to be known in real industrial applications, it is not used to drive the partitioning of the transients into clusters but only to verify the performance of the proposed methodology.

The similarity matrix $\overline{\bar{W}}$ of size $150 \cdot 150$ obtained by computing the similarity measure between all possible pairs of trajectories is shown in Figure 5: the larger the similarity $\mu_{i j}$, the brighter the shade of the $i j$-element of the matrix. 


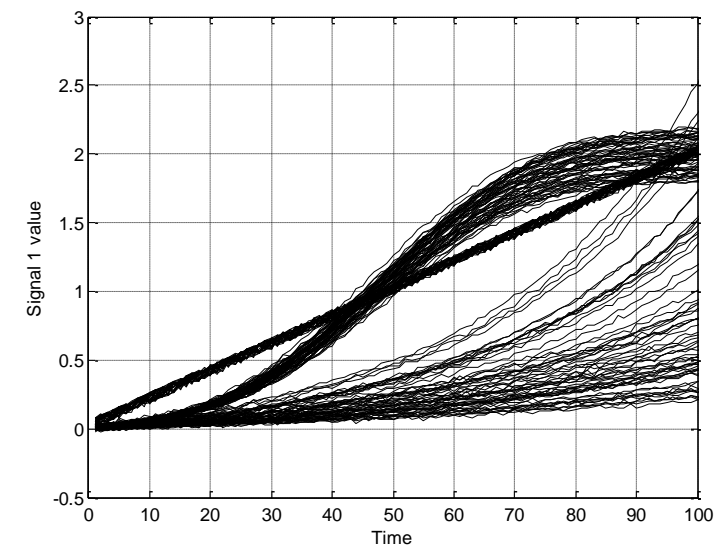

Fig. 1. Projection on the signal 1 axis of the 150 simulated transients.

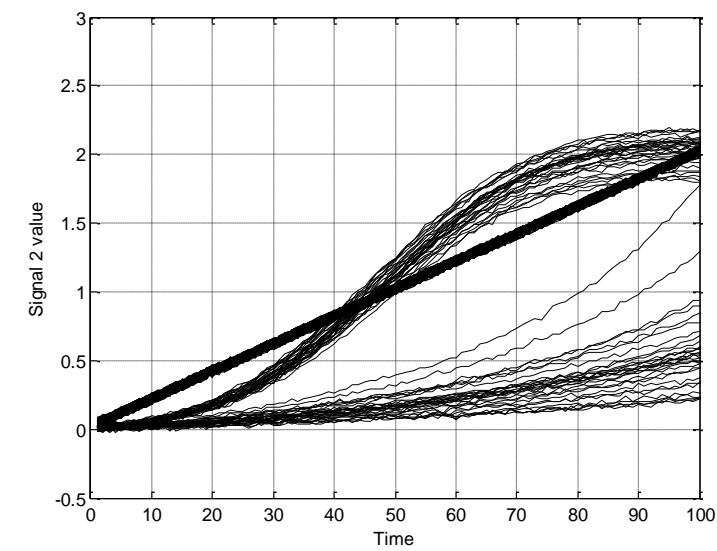

Fig. 2. Projection on the signal 2 axis of the 150 simulated transients.

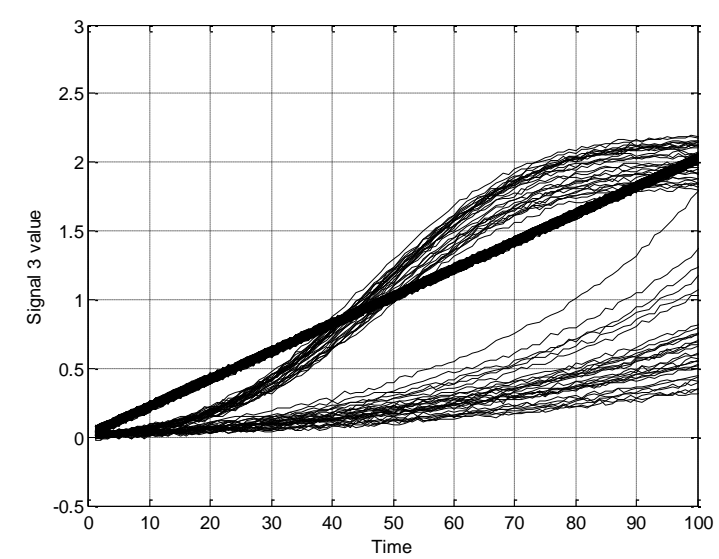

Fig. 3. Projection on the signal 3 axis of the 150 simulated transients.

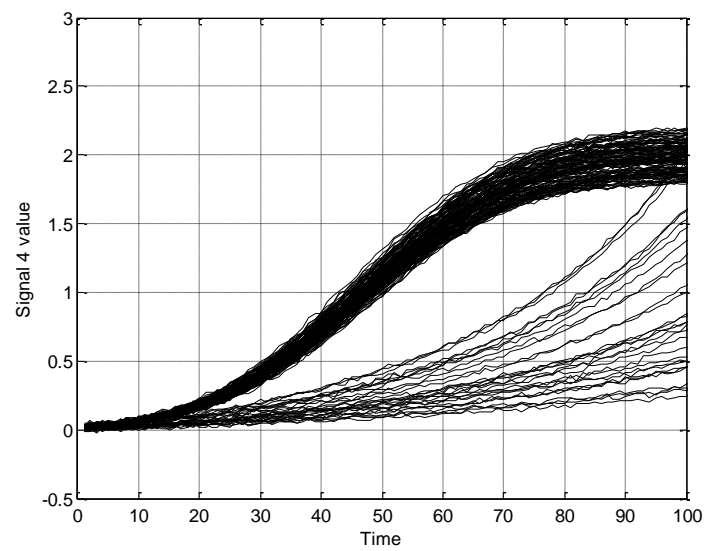

Fig. 4. Projection on the signal 4 axis of the 150 simulated transients.

A large number of edges have large weights $\mu_{i j}$ (i.e., the vertices are strongly connected, which means that the corresponding trajectories are similar), but it is not easy to distinguish a partition of the graph in groups of trajectories. If the trajectories were sorted in such a way that similar trajectories were in consecutive rows of the matrix, e.g., all trajectories of class 1 in rows and columns from 1 to 30 , all trajectories of class 2 in rows and columns from 31 to 60 and so on, the representation of the matrix would lead to a checkboard-like structure [Kluger et al., 2003]. This is due to the fact that if two trajectories are similar between them they tend to have large similarity with the other trajectories and, thus, the corresponding rows and columns in the Figure would have the same sequences of shades. In this sense, from the graphical point of view, the problem of clustering the trajectories may be seen as the problem of finding a proper ordering of the trajectories that leads to the visualization of a checkboard-like structure.

Figure 6 shows the 150 eigenvalues obtained by applying spectral analysis to matrix $\overline{\bar{W}}$, as described in Section 3.2. Since the first five eigenvalues are very close to zero and the sixth is remarkably larger, the number of clusters $C$ is set equal to 5 .

The new representation of the 150 trajectories into the first five dimensions of the eigenvectors space is reported in Figure 7.

In practice, the problem of clustering the 150 trajectories $\overline{\overline{X^{i}}}$ is now reduced to the problem of finding five clusters among the 150 5-dimensional vectors $\bar{t}_{i}$, where each $\overline{t_{i}}$ constitutes a reduced representation of $\overline{\overline{X^{i}}}$. 
The FCM partitioning of the obtained vectors $\bar{t}_{i}$ provides the memberships $m_{i c}$ of the $i$-th transient, $i=1,2, \ldots, 150$, to the $C$ clusters, $\quad c=1,2, \ldots, 5$. Crisp assignment of the vectors to the clusters is then performed by largest membership value.

The trajectories are reordered according to the clusters found: all trajectories assigned to cluster 1 are represented in the first 30 rows, all trajectories of cluster 2 in rows from 31 to 60 , etc... This indeed leads to a checkboard-like structure (Figure 8 ) where the blocks of highly and lowly similar trajectories are easily identifiable [Baraldi et al., 2012b].

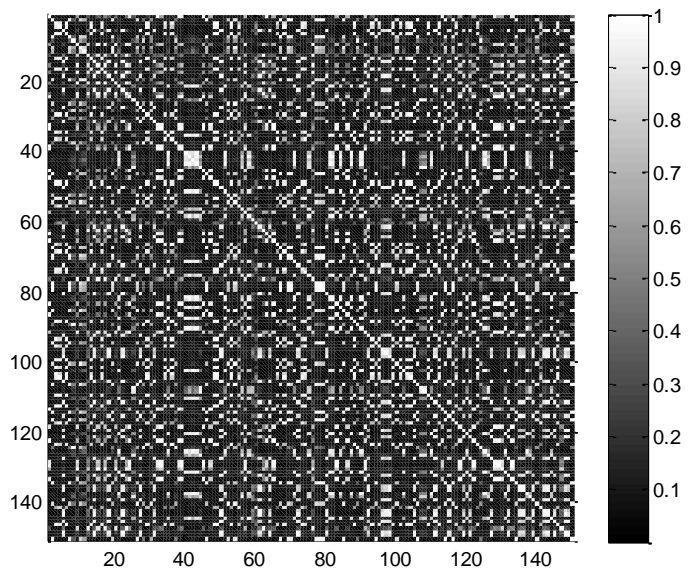

Fig. 5. The similarity matrix $\overline{\bar{W}}$ : the larger the similarity, the brighter the shade of the $i j$-th element.

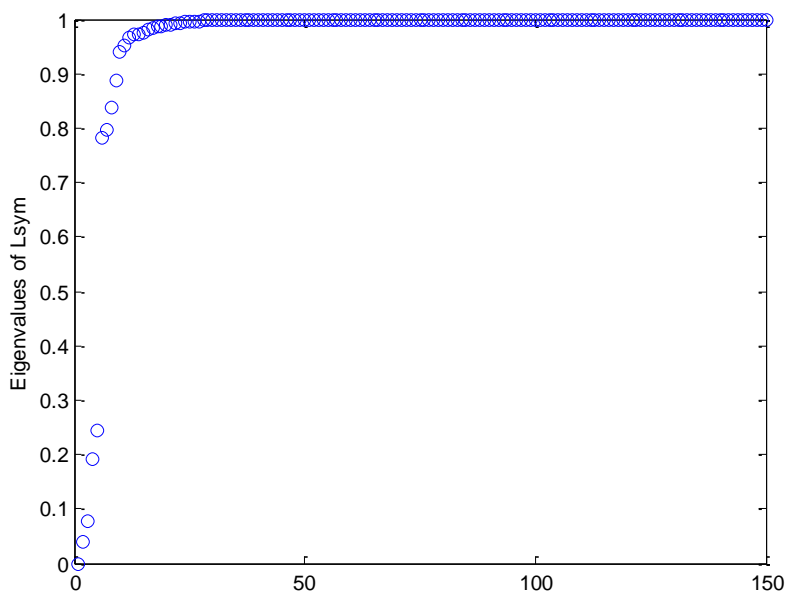

Fig. 6. The 150 eigenvalues of $\overline{\bar{L}}_{s y m}$.

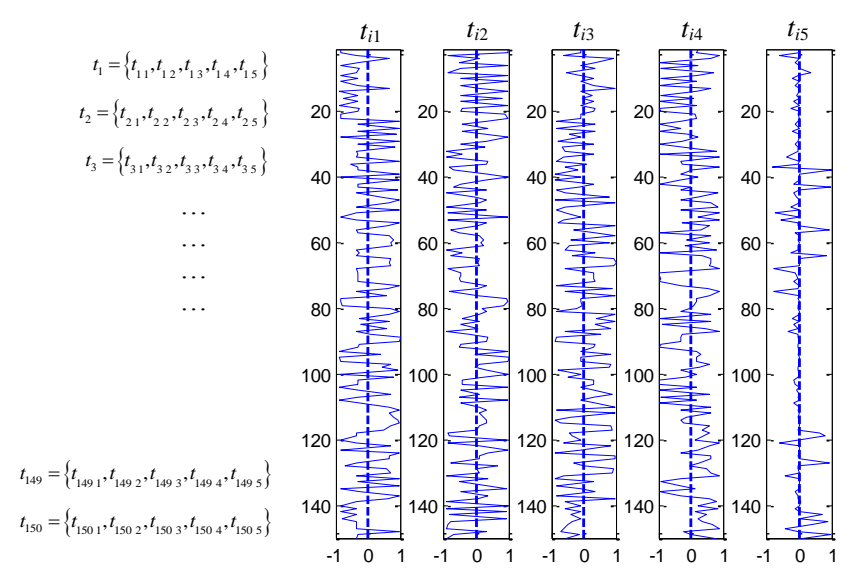

Fig. 7. Representation of matrix $\overline{\bar{T}}$. Columns correspond to the values of the first 5 eigenvectors of $\overline{\bar{L}}_{s y m}$ of the raw data matrix of Figure 5.

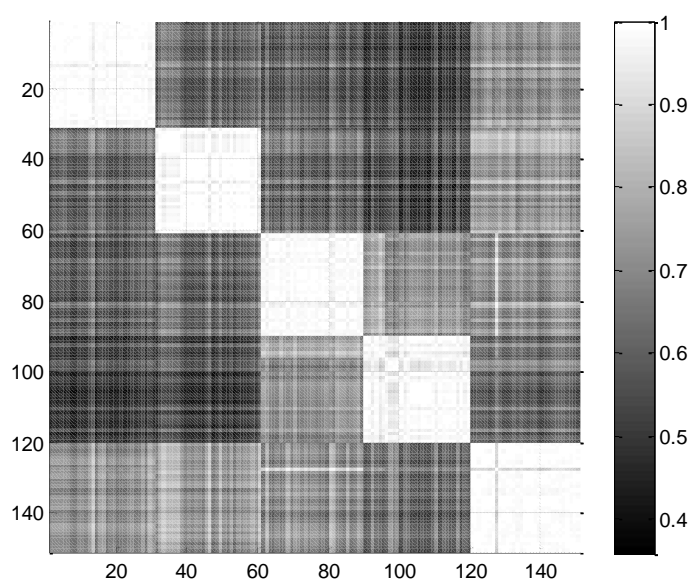

Fig. 8. Shuffled matrix containing checkboard clusters of trajectories.

\section{Fault diagnosis in the pressurizer of a PWR}

A case study regarding a pressurizer of a PWR NPP has been considered. Figure 9 is a schematic representation of the pressurizer system for which a Matlab SIMULINK model has been developed, based on the application of the mass and energy conservation equations to the two regions of vapor and liquid; exchanges between the two regions, due to evaporation of liquid and condensation of steam, are taken into account [Kuridan et al., 1998; Todreas et al., 1990]. The system of nonlinear differential equations describing the model is detailed in [Baraldi et al., 2010].

In order to represent a realistic situation, the simulations have been carried out based on the operational parameters of a standard PWR pressurizer (Table 1). 
Furthermore, the total mass of water entering/exiting the pressurizer during a surge line mass flow transient has been related to the temperature variations of the coolant in the Primary Heat Transport (PHT) system.

In order to test the method on pseudo-realistic data, white noise has been added to each signal according to engineering considerations on the sensors accuracy [Hashemian, 2004; Johnson, 2008]. Table 2 reports the standard deviations of the considered noises.

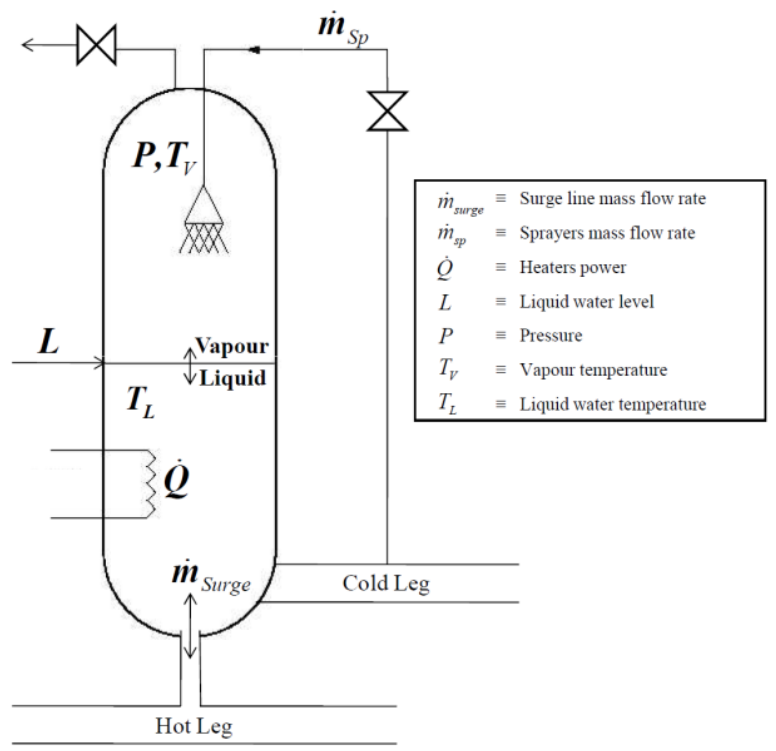

Fig. 9. Simplified model of a pressurizer.

Table 1. Initial conditions of the pressurizer

\begin{tabular}{|c|c|}
\cline { 2 - 2 } \multicolumn{1}{c|}{} & Initial condition \\
\hline Level & $7.22 \mathrm{~m}$ \\
\hline Liquid temperature & $342.1^{\circ} \mathrm{C}$ \\
\hline Vapor temperature & $342.3^{\circ} \mathrm{C}$ \\
\hline Pressure & $150 \mathrm{bar}$ \\
\hline
\end{tabular}

Table 2. Standard deviation of sensors noise

\begin{tabular}{|c|c|}
\cline { 2 - 2 } \multicolumn{1}{c|}{} & Noise standard deviation \\
\hline Level sensor & $\pm 0.01 \mathrm{~m}$ \\
\hline Pressure sensor & $\pm 0.5 \mathrm{bar}$ \\
\hline Flow sensor & $\pm 0.2 \mathrm{~kg} / \mathrm{s}$ \\
\hline Power sensor & $\pm 50 \mathrm{~kW}$ \\
\hline
\end{tabular}

A block diagram of the model identifying the inputs, state of the system, outputs and controller variables is shown in Figure 10. The control of the level $L$ and the pressure $P$ in the pressurizer is achieved through a feedback control scheme which reproduces that used in a standard PWR pressurizer. According to the control scheme illustrated in [Baraldi et al., 2010], the pressure $f_{P}$ and level $f_{L}$ are the controlled signals as well as the controller input signals; the sprayers mass flow rate $\dot{m}_{s p}$, and the heaters power $\dot{Q}$ are the controller outputs. The present case study focuses on some faults which can occur to the pressurizer control system and can lead to undesired behaviors of the pressurizer. In particular, three different classes of faults are taken into account (Table 3):

1) heaters fail stuck with a fixed power output value $\dot{Q}$;

2) sprayers fail stuck with a fixed mass flow rate $\dot{m}_{s p}$;

3) the communication between the controller and actuators fails: sprayers and heaters receive from the controller the command to provide a wrong quantity of water and heat, respectively.

Notice that these fault states can be identified only during plant transients characterized by an in/out-surge mass flow which requires the operation of the control system components, whereas they are latent in case of stationary operations of the plant.

To reproduce the pressurizer behavior in case of normal and faulty conditions, $N=80$ transients have been simulated with a time horizon of $1200 \mathrm{~s}$. In all the transients considered, the total out-surge flow, $m_{\text {surge }}$, is of $1444 \mathrm{~kg}$ with a variable flow rate $\dot{m}_{\text {surge }}$ in the range of $[-11 ;-5] \mathrm{kg} / \mathrm{s}$. The initial state of the pressurizer is characterized by the parameter values reported in Table 1. The simulated transients are 20 in normal conditions and 20 for each of the three classes of faults. The onset of the fault occurs at a random time $t_{f}$ sampled from a uniform distribution between $30 \mathrm{~s}$ and the time at which the out-surge mass flow stops. In case of a class 3 fault, the actuators receive from the controller the command of providing a quantity of water or heat proportional to the correct one multiplied by a random factor, $b$, sampled from a uniform distribution [0;2]. Notice that in the case here considered of out-surge transients during which sprayers are not called in operation, class 2 faults are not distinguishable from normal condition transients.

Table 3. Standard deviation of sensors noise

\begin{tabular}{|c|c|}
\hline Class $\mathbf{1}$ & Heaters fail stuck with a fixed power output value $\dot{Q}$ \\
\hline Class $\mathbf{2}$ & Sprayers fail stuck with a fixed mass flow rate $\dot{m}_{s p}$ \\
\hline Class $\mathbf{3}$ & Communication between the controller and actuators fails \\
\hline $\begin{array}{c}\text { Normal } \\
\text { conditions }\end{array}$ & $\begin{array}{c}\text { Heaters, sprayers and communication between controller } \\
\text { and actuators work in normal conditions }\end{array}$ \\
\hline
\end{tabular}




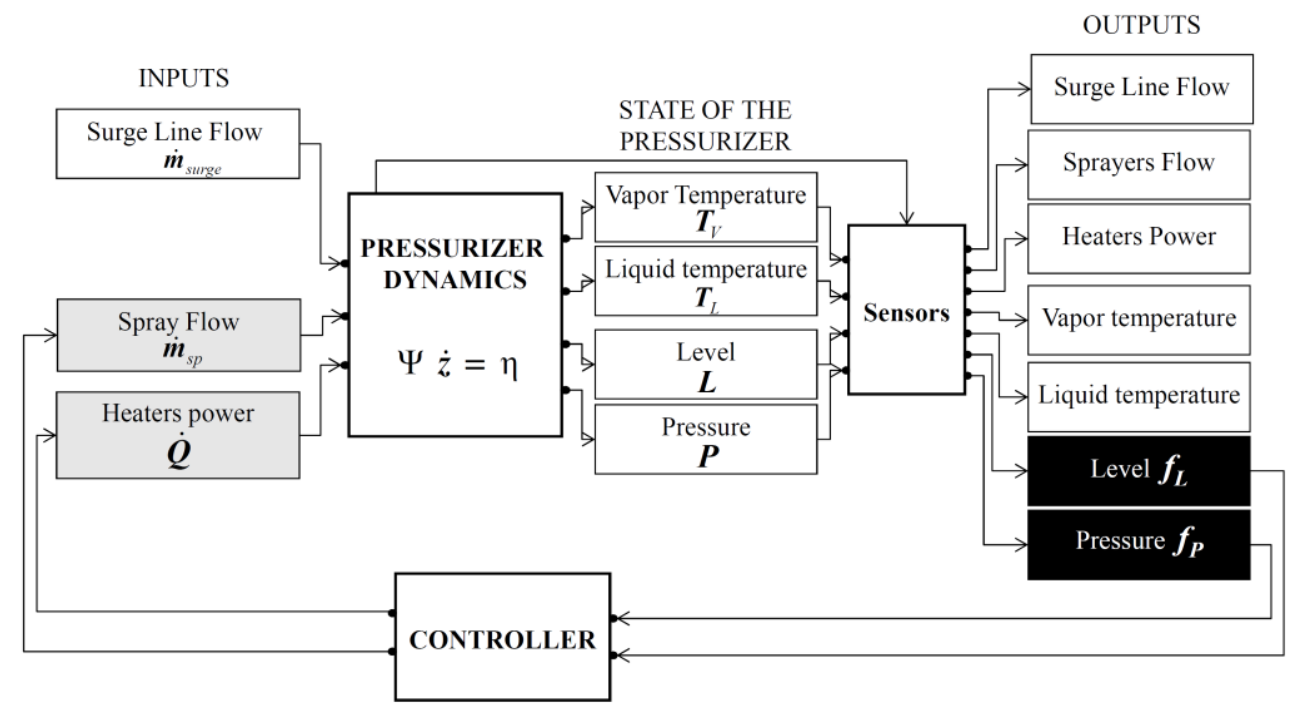

Fig. 10. Inputs, state, controller outputs (gray), controller inputs (black) and sensors outputs of the SIMULINK model of the pressurizer.

As an example, Figures 11-17 show the consequences of a class 1 fault (i.e., heaters fail stuck with a fixed power output value $\dot{Q}$ ) on the behavior of the pressurizer. The considered fault consists in a blockage of the heaters at time $t=78 \mathrm{~s}$ during the out-surge transient of Figure 11 characterized by a surge mass flow rate of $-9.51 \mathrm{Kg} / \mathrm{s}$. The out-surge flow produces a reduction in the pressure, $P$, and liquid and steam temperatures, $T_{L}$ and $T_{V}$, which induces the controller to turn on the heaters (Figure 13). In case of a nominal transient (black continuous line in Figure 15), the pressure promptly starts increasing and after $1000 \mathrm{~s}$ reaches the desired value, whereas in the case of faulty transient, due to the reduced power provided by the heaters, the pressure recovery is slower and after $1200 \mathrm{~s}$ its value is still 1 bar lower than the required (gray dotted line in Figure 15).

The signals $L, P, T_{L}$ and $T_{V}$ measured during the transients are considered for the clustering. Thus, each transient is represented by a $Z=4$ dimensional trajectory. As in the previous case study, the information on the true class of the trajectory and on the total number of classes of faults causing the 80 transients is not used to drive the grouping of the transients, but only to verify the performance of the proposed methodology.

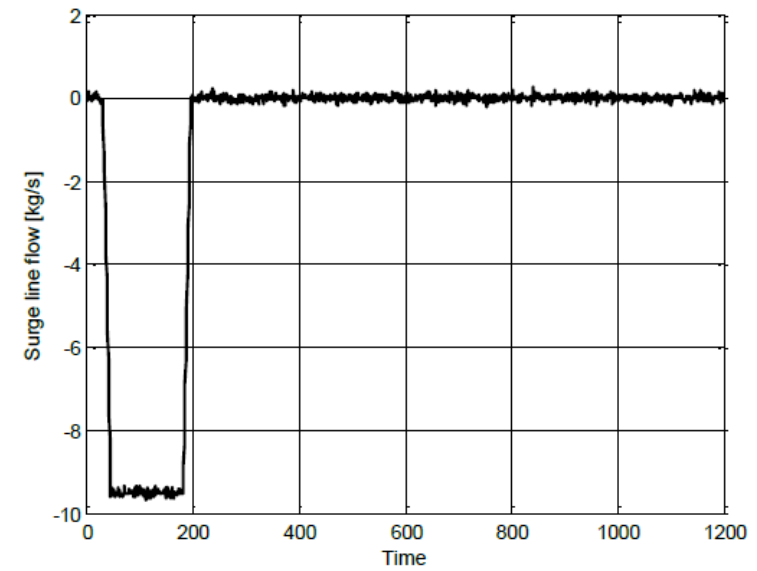

Fig. 11. Evolution of the surge line flow rate.

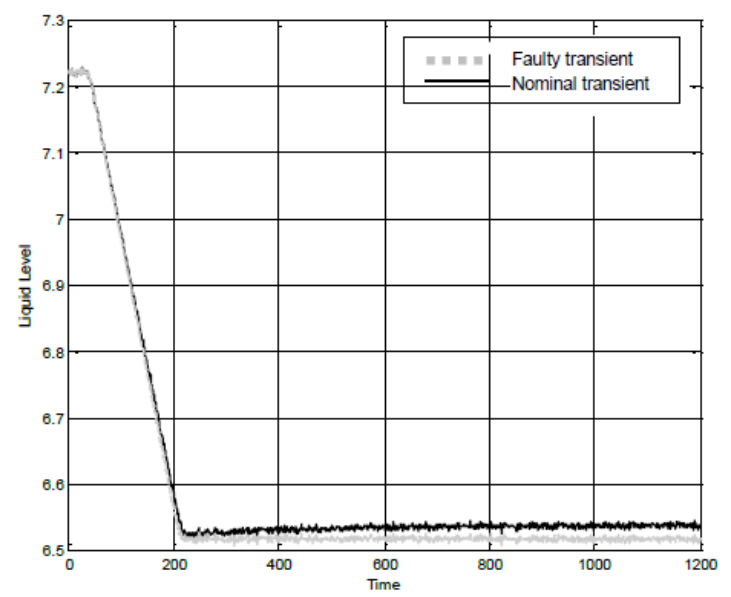

Fig. 12. Evolution of the liquid level for a nominal transient and the faulty transient. 


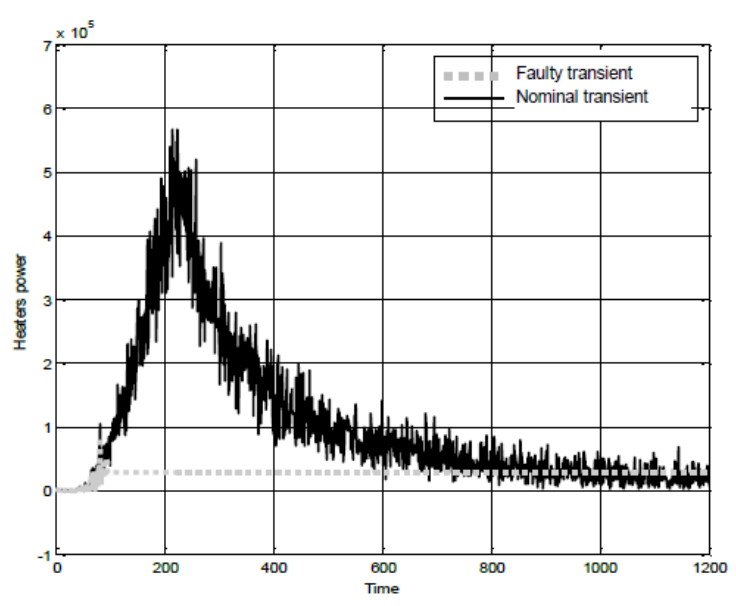

Fig. 13. Evolution of the heaters power for a nominal transient and the faulty transient.

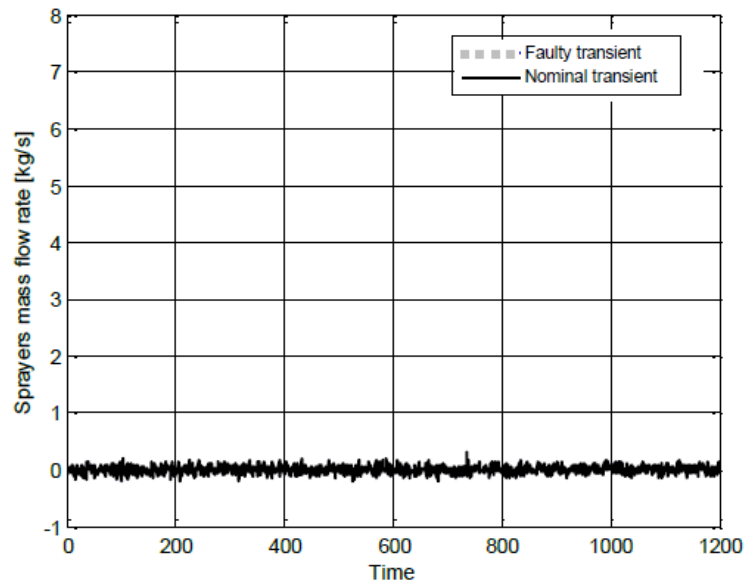

Fig. 14. Evolution of the sprayers mass flow rate.

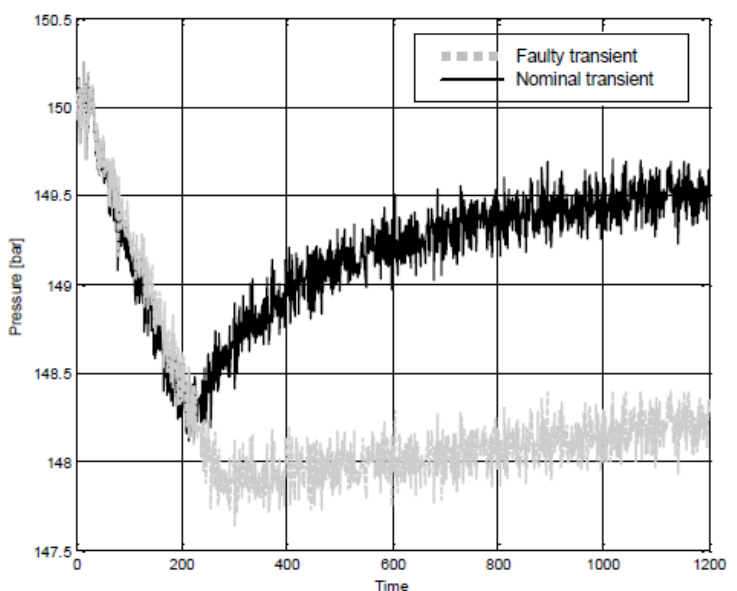

Fig. 15. Evolution of the pressure for a nominal transient and the faulty transient.

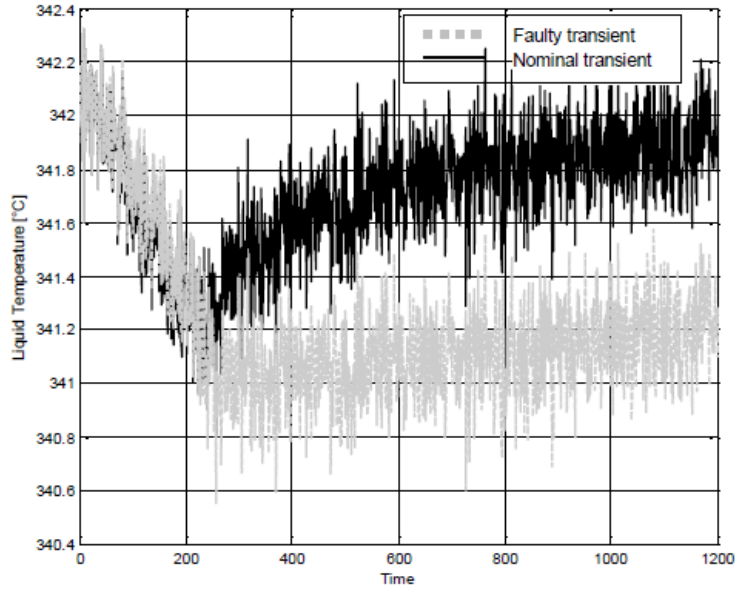

Fig. 16. Evolution of the liquid temperature for a nominal transient and the faulty transient.

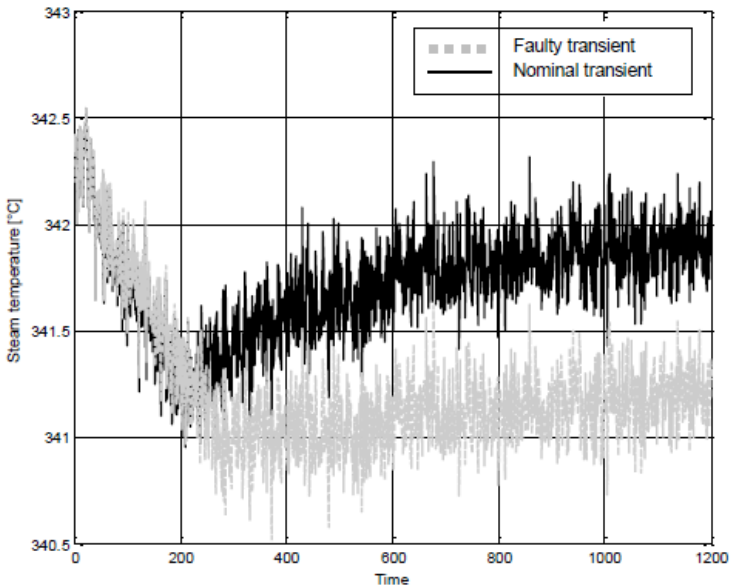

Fig. 17. Evolution of the steam temperature for a nominal transient and the faulty transient.

The application of the methodology described in Section 3 leads to the identification of $C=4$ groups of transients. Figures 18-21 show the evolution of the pressure in all the transients of clusters 1-4, respectively. Clusters 1, 2 and 4 appear to be very compact since the signal numerical values of the transients belonging to the same cluster are very similar and apparently show similar form, slope and curvature of the signal trajectories. On the other hand, cluster 3 is characterized by different numerical values of the signals in the different transients but all trajectories are characterized by a linear increase of the pressure which allows distinguishing them from the other clusters trajectories. This shows that, in this case, the 
methodology has been able to identify a functional similarity between the trajectories.

In practical industrial cases where the information on the true class of the transients is not available, understanding and interpretation of the physical cause originating the onset of the transients (fault class) can be sought by analyzing the signal evolutions in the trajectories of the cluster. To this aim, instead of analyzing all trajectories in a cluster, experts can focus on a most representative trajectory, i.e., the prototypical trajectory with largest membership value to the cluster. In this respect, Figure 22 shows the evolutions of the 4 monitored signals for the prototypes of the 4 clusters identified. Experts may recognize that the behavior of the signals in the prototype trajectory of cluster 4 is very similar to that in case of normal conditions. Furthermore, the slow linear increase of the pressure and temperature signal in the prototypical transient of cluster 3 suggests that the heaters are providing an insufficient power, i.e., a class 1 fault as previously described (Table 3). Cluster 1 trajectory is characterized by a slightly faster increase of the pressure than in normal conditions: this should lead the expert to identifying a class 3 fault caused by the heaters providing higher power than required, i.e., a class 1 fault with $b>1$. Finally, cluster 2 is characterized by a slightly slower increase of temperature and pressure than that in nominal case: a possible cause can be a lower power provided by the heaters, as in class 3 fault with $b<1$.

Using the information on the true class of the simulated transients, we can verify the correspondence between clusters and fault classes. Table 4 reports the number of transients of each fault class contained in the 4 clusters obtained. Notice that both clusters 1 and 2 are formed by transients of class 3 (communication between the controller and actuators fails): the former consists in faulty transients caused by heaters providing more power than necessary ( $b>1$, Figure 23), the latter by less power than necessary $(b<1$, Figure 24). All the transients of class 1 (heathers fail stuck at a fixed power) are contained in cluster 3 , together with 3 transients of class 3 characterized by a very large reduction of the power provided by the heaters $(b \ll 1$, Figure 25). Finally cluster 4 contains all the transients in normal conditions, fault class 2 transients and 5 transients of class 1 characterized by values of $b \approx 1$ (Figure 26). The merging of the normal condition and class 2 transients into the same cluster depends on the fact that transients of class 2 , i.e., due to a failure of the sprayers, are not distinguishable from the nominal condition transients in the case here considered of outsurge transients. In fact, in this case, sprayers are never called in operation and, therefore, their failure cannot affect the transient functional behaviour. Furthermore, transients of class 1 characterized by values of $b \approx 1$ are those in which the actuators receive from the controller the command of providing a quantity of water or heat practically equal to the correct one and, therefore, show the same functional behaviour of the normal condition transients. These results show that the methodology is capable of distinguishing transients characterized by similar functional behaviour of the signals.

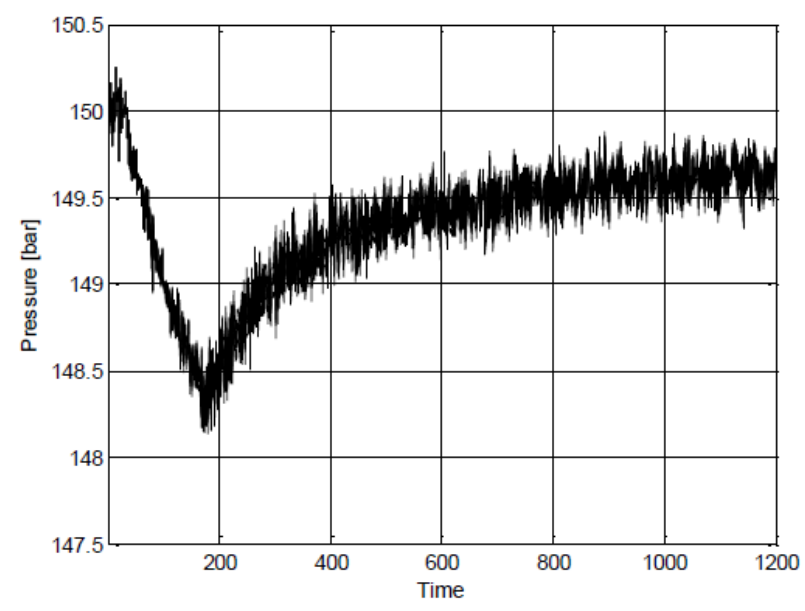

Fig. 18. Pressure profiles of trajectories belonging to cluster 1 .

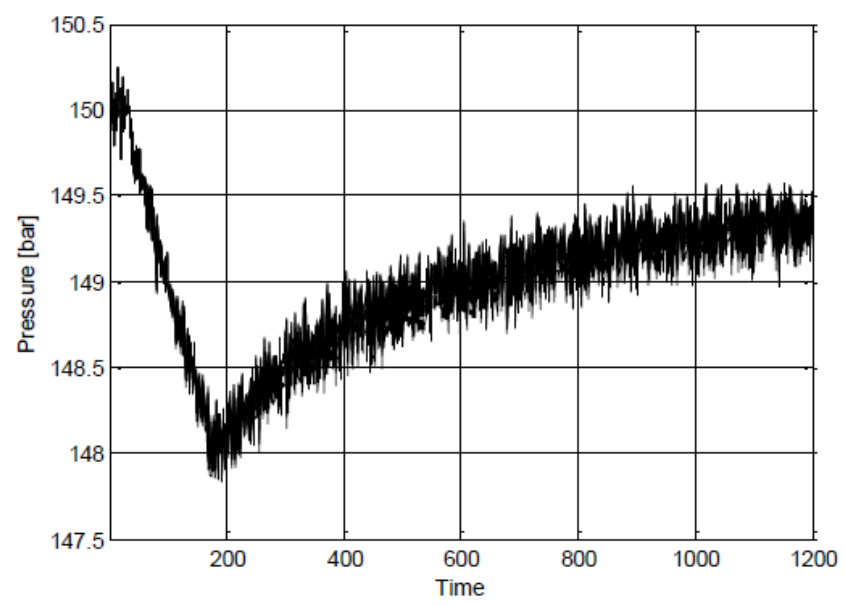

Fig. 19. Pressure profiles of trajectories belonging to cluster 2 . 


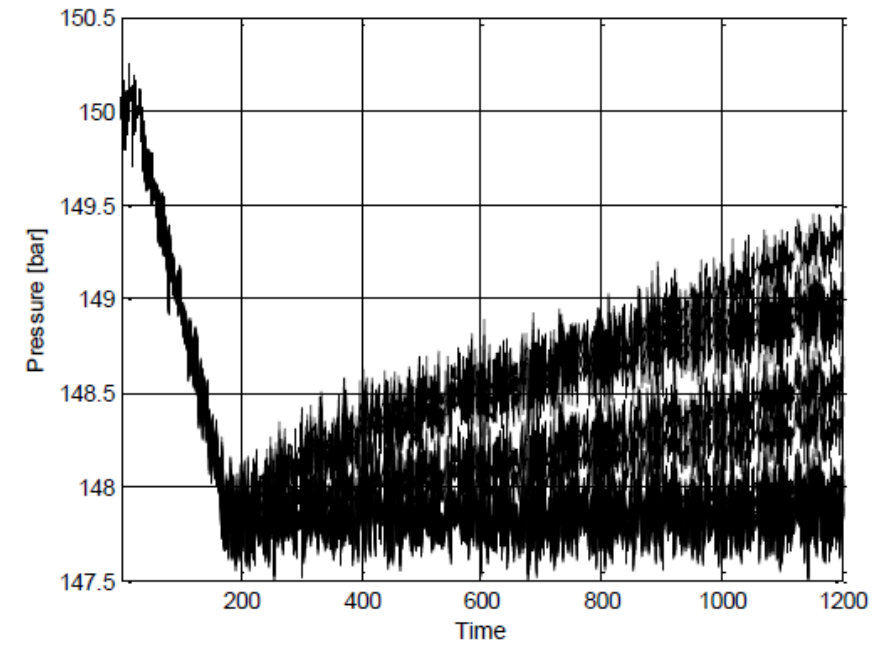

Fig. 20. Pressure profiles of trajectories belonging to cluster 3 .

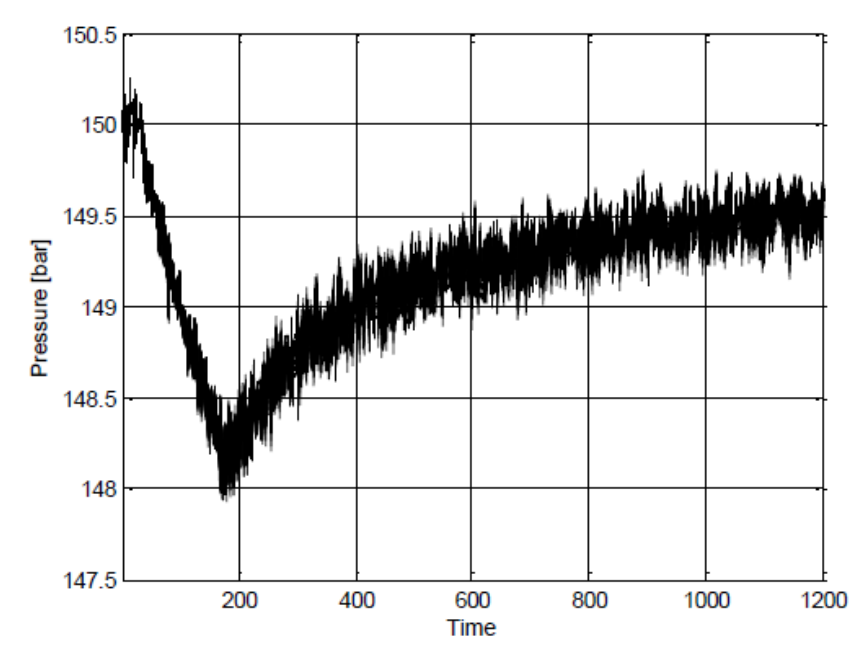

Fig. 21. Pressure profiles of trajectories belonging to cluster 4 .
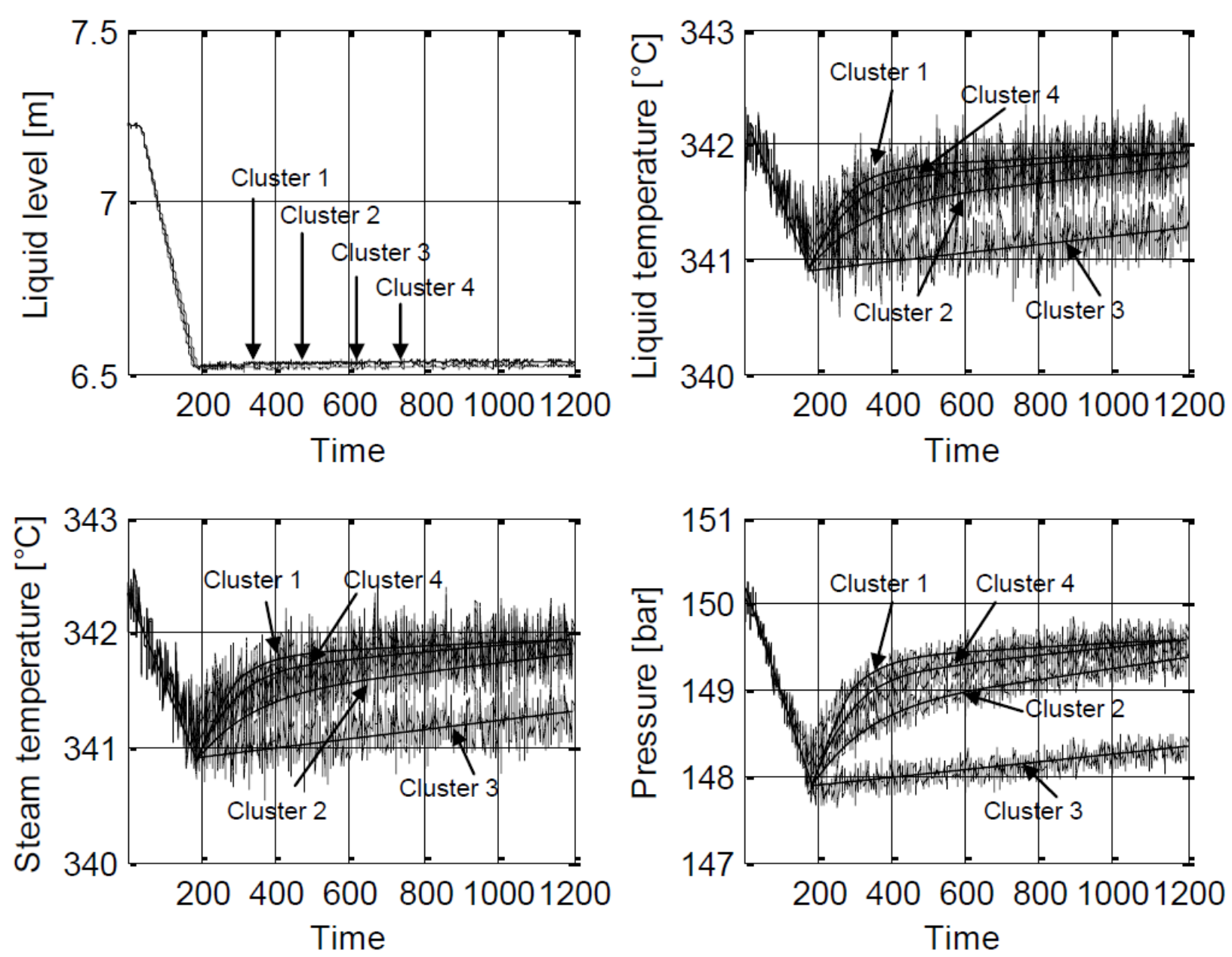

Fig. 22. Prototypical trajectories (noisy thin line) and filtered signals (smooth thick line). 
Table 4. Performance of the clustering approach

\begin{tabular}{|l|c|c|c|c|}
\cline { 2 - 5 } \multicolumn{1}{c|}{} & Class 1 & Class 2 & Class 3 & $\begin{array}{c}\text { Normal } \\
\text { condition }\end{array}$ \\
\hline Cluster 1 & - & - & 5 & - \\
\hline Cluster 2 & - & - & 7 & - \\
\hline Cluster 3 & 20 & - & 3 & - \\
\hline Cluster 4 & - & 20 & 5 & 20 \\
\hline
\end{tabular}

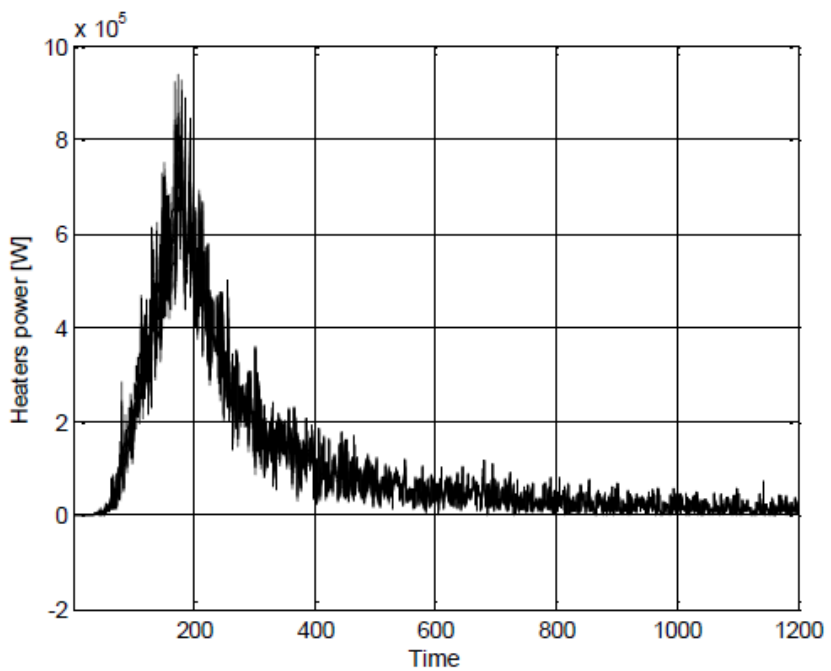

Fig. 23. Heaters power profile of trajectories belonging to cluster 1 .

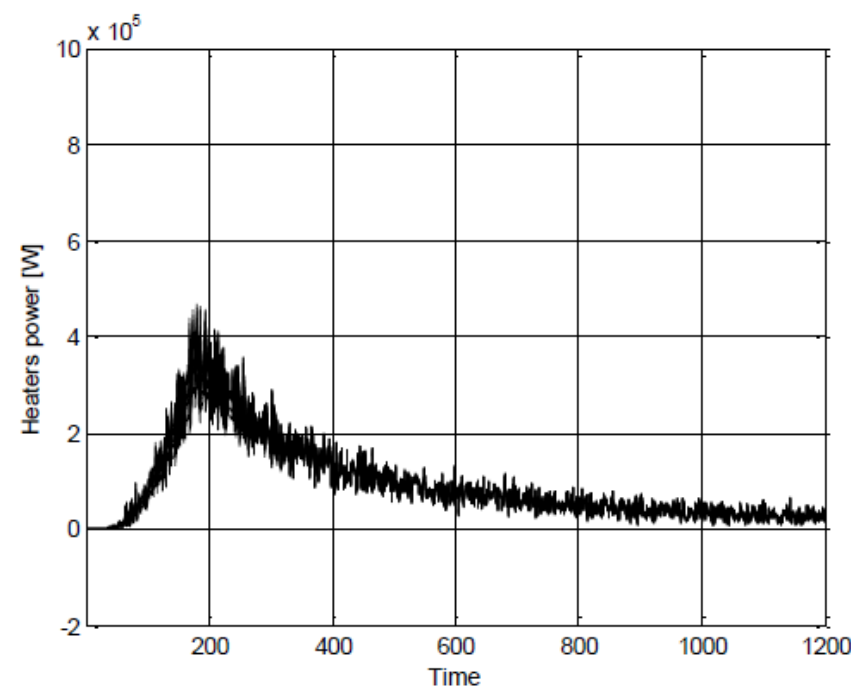

Fig. 24. Heaters power profile of trajectories belonging to cluster 2 .

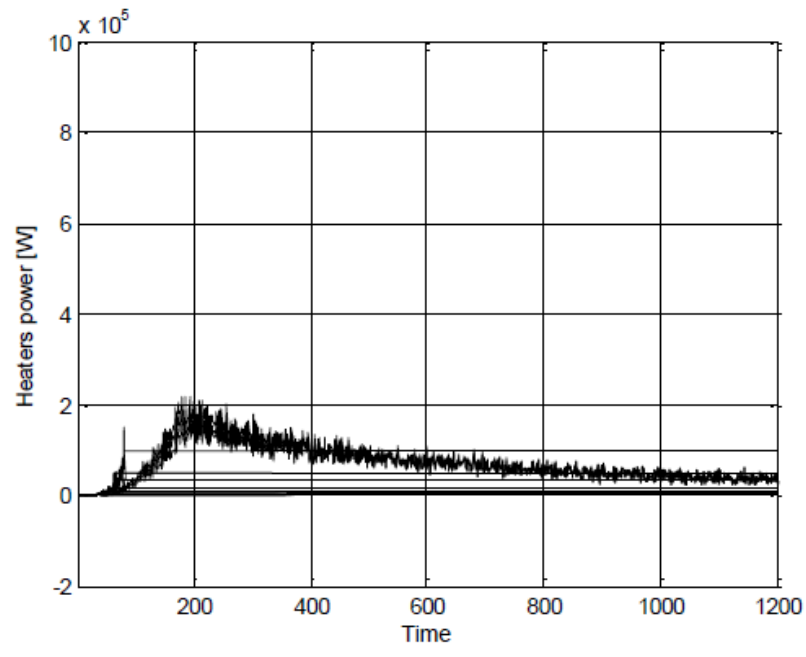

Fig. 25. Heaters power profile of trajectories belonging to cluster 3 .

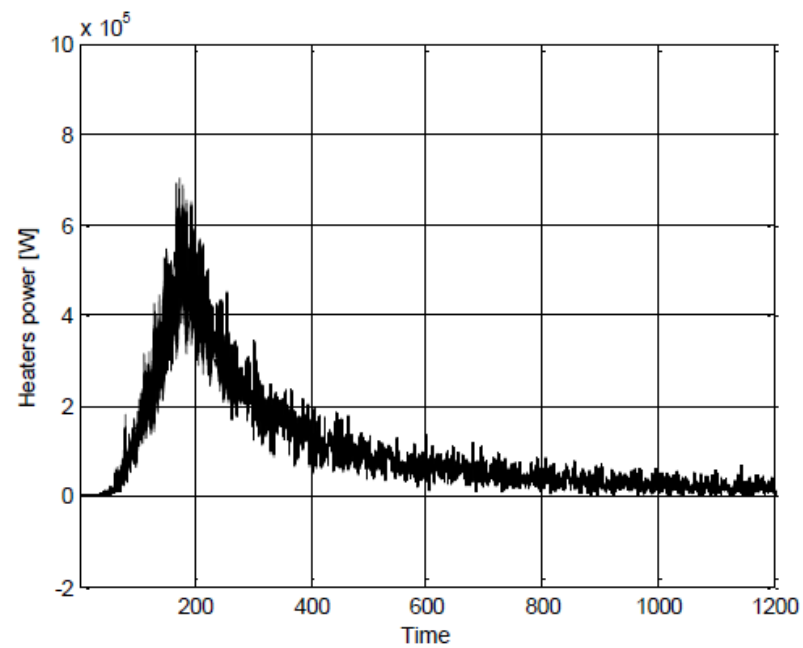

Fig. 26. Heaters power profile of trajectories belonging to cluster 4.

\section{Conclusions}

We have developed a methodology for the identification of groups of transients with similar behaviour because originated by faults of the same type. We have combined Haar wavelets transform, fuzzy similarity, spectral analysis and Fuzzy C-Means clustering. We have shown applications to an artificial case study and to the identification of transients in the pressurizer of a PWR.

The main conclusions of the analysis are:

1) Haar wavelets allow capturing the functional behavior, i.e., the shape of the transient; 
2) spectral analysis allows identifying the number of clusters of similar trajectories and extracting their most relevant features;

3) the FCM algorithm allows finding the clusters of similar transients and identifying a prototypical trajectory for each cluster, which can then be used for fault understanding and interpretation.

A drawback of the methodology is that if new transients become available, it is not possible to dynamically update the clustering of the trajectories, but we have to repeat spectral analysis and FCM clustering on the new similarity matrix extended to contain the fuzzy similarity between all the new and old trajectories. To overcome this limitation, in the future we intend to look at an incremental learning framework.

The continuation of this work will also consider the application to real datasets collected during NPP operation and the development of an empirical classification scheme based on a supervised technique which exploits the cluster results gained from the analysis here presented.

\section{Acknowledgements}

This research has been carried out under contract EDF/PDM C935C50010 "Processing condition monitoring data for diagnosis and prognosis of components of electricity production plants" funded by EdF R\&D.

\section{References}

[Alata et al., 2008] M. Alata, M. Molhim, and A. Ramini, "Optimizing of Fuzzy C-Means Clustering Algorithm Using GA", World Academy of Science, Engineering and Technology, p.224-229, 2008.

[Alpert et al., 1999] C. Alpert, A. Kahng, S. Yao, "Spectral partitioning: the more eigenvectors, the better", Discrete Applied Math, Volume 90, pages 3-26, 1999.

[Angstenberger, 2001] L. Angstenberger, "Dynamic fuzzy pattern recognition, international series in intelligent technologies", vol. 17. Dordrecht: Kluwer Academic Publishers; 2001.

[Baraldi et al., 2010] P. Baraldi, A. Cammi, F. Mangili, E. Zio, "An ensemble approach to sensor fault detection and signal reconstruction for nuclear system control", Annals of Nuclear Energy 37 (2010) 778-790.

[Baraldi et al., 2012a] P. Baraldi, F. Di Maio, L. Pappaglione, E. Zio, R. Seraoui, "Condition Monitoring of Electrical Power Plant Components During Operational Transients", Proceedings of the Institution of Mechanical Engineers, Part O, Journal of Risk and Reliability, 226(6) 568-583, 2012.

[Baraldi et al., 2012b] P. Baraldi, F. Di Maio, E. Zio "Unsupervised Clustering for Fault Diagnosis", proceedings of Prognostics and System Health Management Conference (PHM-2012), Beijing, China, 23-25 May 2012.

[Bezdek, 1981] J.C., Bezdek, "Pattern Recognition with Fuzzy Objective Function Algorithms", Plenum, New York, 1981.

[Cheon et al., 1993] Cheon, S. W., Chang, S. H., Chung, H. Y., Bien, Z. N., Application of neural networks to multiple alarm processing and diagnosis in nuclear power plants, IEEE Transactions on Nuclear Science 40 (1) , pp. 11-20, 1993.

[D'Antone, 1992] D'Antone, I., A neural network in an expert diagnostic system, IEEE Transactions on Nuclear Science 39 (2 pt 1), pp. 58-62, 1992.

[Di Maio et al., 2011] F. Di Maio, P. Secchi, S. Vantini, E. Zio, "Fuzzy C-Means Clustering of Signal Functional Principal Components for Post-Processing Dynamic Scenarios of a Nuclear Power Plant Digital Instrumentation and Control System", IEEE - Transactions on Reliability, Volume 60, Issue 2, June 2011, pages 415-425.

[Dubois et al., 1988] D. Dubois, H., Prade, C., Testemale, "Weighted Fuzzy Pattern Matching", Fuzzy Sets and Systems, 28, 1988, p. 313-331, 1988.

[Hasemian, 2004] Hashemian, H.M., 2004. Sensor Performance and Reliability, ISA.

[Ikonomopoulos et al., 1998] Ikonomopoulos, A., Endou, A., Wavelet decomposition and radial basis function networks for system monitoring, IEEE Transactions on Nuclear Science 45 (5), pp. 2293-2301, 1998.

[Joentgen et al., 1999] A. Joentgen, L. Mikenina, R. Weber, H.J. Zimmermann, “ Dynamic Fuzzy Data Analysis based on Similarity between Functions", Fuzzy Sets and Systems, 105 (1), 1999, p. 81-90, 1999.

[Johnson, 2008] Johnson, F., 2008. Nuclear Reactor Controls and Instrumentation. Wexford College Press.

[Kim et al., 1996] Kim, K., Bartlett, E.B., Nuclear power plant fault diagnosis using neural networks with error estimation by series association, IEEE Transactions on Nuclear Science 43 (4 PART 2), pp. 2373-2388, 1996.

[Klir and Yuan, 1995] Klir, G., Yuan, B., 1995. Fuzzy Sets and Fuzzy Logic: Theory and Application. Prentice Hall, Englewood Cliffs, NJ.

[Kluger et al., 2003] Y. Kluger, R. Basri, J.T. Chang. M. Gerstein, "Spectral Biclustering of Microarray Data: Coclustering Genes and Conditions", Genome Research, Volume 13, pages 703-716, 2003.

[Kuridan et al., 1998] Kuridan, R.M., Beynon, T.D., 1998. A linearized non steady model for the pressurizer of the safe integral reactor concept. Progress in Nuclear Energy 33.

[Leguizamon et al., 1996] S. Leguizamon, H. P. and S. Azzali, "Unsupervised Fuzzy C-Means Classification for the Determination of Dynamically Homogeneous Areas", p.851-856, 1996.

[Mohar, 1997] B. Mohar, "Some Applications of Laplace Eigenvalues of Graphs", in G. Hahm and G. Sabidussi (Eds.), Graph Symmetry: Algebraic Methods and Applications (Vol. NATO ASI Ser. C 497), pages 225-275, 1997.

[Na et al., 2004] Na, M.G., Shin, S.H., Lee, S.M., Jung, D.W., Kim, S.P., Jeong, J.H., Lee, B.C., Prediction of major transient scenarios for severe accidents of nuclear power plants, IEEE Transactions on Nuclear Science 51 (2), pp. 313-321, 2004. 
[Ogden, 1997] R. T. Ogden, "Essential Wavelets for Statistical Applications and Data Analysis", Birkhausen, Boston, 1997.

[Reifman, 1997] Reifman, J., 1997. Survey of artificial intelligence methods for detection and identification of component faults in nuclear power plants. Nucl. Technol. $119,76-97$.

[Roverso, 2000] D. Roverso, "Soft computing tools for transient classification", Information Sciences, 127, pp 137$156,2000$.

[Roverso, 2003] D. Roverso, "Fault diagnosis with the aladdin transient classifier." In: Proceedings of System Diagnosis and Prognosis: Security and Condition Monitoring Issues III, AeroSense2003, Aerospace and Defense Sensing and Control Technologies Symposium. Orlando, FL, 21-25 April 2003.

[Sheng et al., 2004] Sheng, Y., Rovnyak, S.M., 2004, Decision Tree-Based Methodology for High Impedance Fault Detection, IEEE Transactions on Power Delivery, Vol. 19, No. 2.

[Strang et al., 1996] G. Strang, T. Nguyen, Wavelets and Filter Banks, Wellesley-Cambridge, 1996

[Todreas et al., 1990] Todreas, N., Kazimi, M.S., 1990. Nuclear Systems 1. Taylor \& Francis.

[Venkatasubramanian et al., 2003] Venkatasubramanian, V., Rengaswamy, R., Yin, K., Kavuri, S.N., 2003, A Review of Process Fault Detection and Diagnosis: Part I: Quantitative Model-based Methods, Computers \& Chemical Engineering, Vol. 27, pp. 293-311.

[von Luxburg, 2007] U. von Luxburg, "A Tutorial on Spectral Clustering", Statistics and Computing, Volume 17(4), pages395-416, 2007.

[Yang, 1993] Yang, M., 1993. A survey of fuzzy clustering. Math. Comput. Modell. 18 (11), 1-16.

[Zadeh, 1965] Zadeh, L., 1965. Fuzzy sets. Inform. Control 8, 338-353.

[Zhao et al., 2007] Zheng Zhao , Huan Liu, "Spectral feature selection for supervised and unsupervised learning", Proceedings of the 24th international conference on Machine learning, p.1151-1157, June 20-24, 2007, Corvalis, Oregon

[Zio et al., 2006a] Zio, E., Gola, G., 2006, Neuro-fuzzy Pattern Classification for Fault Diagnosis in Nuclear Components, Annals of Nuclear Energy, Vol. 33, Issue 5, pp. 415-426.

[Zio et al., 2006b] Zio, E., Baraldi, P., Pedroni, N., Selecting features for nuclear transients classification by means of genetic algorithms, IEEE Transactions on Nuclear Science 53 (3) , art. no. 1645062 , pp. 1479-1493, 2006.

[Zio, 2007] Zio, E., 2007, Soft Computing Methods Applied to Condition Monitoring and Fault Diagnosis for Maintenance, Reliability: Theory and Applications, Vol. 3.

[Zio et al., 2008] Zio, E., Baraldi, P., Popescu, I., From Fuzzy Clustering to a Fuzzy Rule-Based Fault Classification Model, International Journal of Computational Intelligence Systems, Vol. 1, No. 1, 60-76, 2008.

[Zio et al., 2010] Zio, E., Di Maio, F., Stasi, M., A Datadriven Approach for Predicting Failure Scenarios in Nuclear Systems, Annals of Nuclear Energy, 37, 482-491, 2010.

[Zio et al., 2012] E. Zio, F. Di Maio, "Fault Diagnosis and Failure Mode Estimation by a Data-Driven Fuzzy Similarity
Approach", International Journal of Performability Engineering, Vol. 8, No.1, January 2012, pp. 49-66 